\title{
Sodium-Glucose Cotransporter 2 Inhibitors for Heart Failure
}

\author{
Hidekatsu Yanai
}

In this issue (P94), Kutoh reports type 2 diabetic patients who showed changes in cardiac function which is concomitant with changes in ketone bodies by using one of sodium glucose cotransporter 2 inhibitors (SGLT2i), tofogliflozin [1], proposing a possible mechanism for SGLT2i-mediated prevention of heart failure. EMPA-REG OUTCOME trial showed a striking relative risk reduction in hospitalization for heart failure $(35 \%)$ [2]. Further, the CVD-REAL Study, the large multinational study, concluded that treatment with SGLT2 $\mathrm{i}$ was associated with a lower risk of hospitalization for heart failure and death as compared with other glucose lowering drugs, suggesting that the benefits seen in empagliflozin in a randomized trial may be a class effect applicable to a broad population of type 2 diabetic patients in real-world practice [3]. Such attractive studies urge clinicians and scientists to study to elucidate SGLT2i-mediated cardioprotective mechanisms.

Dapagliflozin successfully prevented the development of hypertrophic cardiomyopathy in a diabetic lipodystrophic mouse model [4]. Left ventricular hypertrophy and cardiac diastolic function in a female rodent model of diabetes were improved by empagliflozin [5]. Various factors such as weight loss, blood pressure lowering and sodium depletion, renal hemodynamic effects, effects on myocardial energetics and neurohormonal effects have been suggested to contribute to cardioprotective mechanisms of SGLT2i [6].

SGLT2i may induce relative glucose deficiency, and then may trigger increased lipolysis and fatty acid oxidation which increase hepatic ketone body production [7]. SGLT2 is expressed in pancreatic $\alpha$ cells, and SGLT2 $\mathrm{i}$ promote glucagon secretion [8]. Increased glucagon secretion due to SGLT2i may be also associated with elevation of hepatic ketone body production.

The report by Kutoh focused on the contribution of change in ketone bodies due to SGLT2i to cardioprotection [1]. Ferrannini et al hypothesized that under conditions of persistent mild hyperketonemia during treatment with SGLT2i, $\beta$-hydroxybutyrate is freely taken up by the heart and oxidized in preference to fatty acids, and this fuel selection improves the transduction of oxygen consumption into work efficiency at the mitochondrial level as a "Thrifty Substrate Hypoth-

Manuscript submitted May 25, 2017, accepted June 5, 2017

Department of Internal Medicine, National Center for Global Health and Medicine Kohnodai Hospital, 1-7-1 Kohnodai, Ichikawa, Chiba 272-8516, Japan. Email: dyanai@hospk.ncgm.go.jp

doi: https://doi.org/10.14740/jem426w esis" [9]. Martens et al also suggested that SGLT2i might improve the efficiency of myocardial energetics by offering $\beta$-hydroxybutyrate as an attractive fuel for oxidation [10]. Mudaliar et al postulated that the cardiorenal benefits of SGLT2 $\mathrm{i}$ are due to a shift in myocardial and renal fuel metabolism away from fat and glucose oxidation, which are energy inefficient in diabetic heart and kidney, toward an energy-efficient super fuel like ketone bodies, which improve myocardial/renal work efficiency and function as a "Unifying Hypothesis" [11]. These findings were supported by changes in cardiac function which is concomitant with changes in ketone bodies by SGLT2 $\mathrm{i}$ observed in the report by Kutoh [1].

Bedi et al reported increased ketone utilization in the severely failing human heart independent of diabetes, supporting that ketone body is an alternative fuel due to a crucial adaptation in the failing heart [12]. Nagao et al showed that $\beta$-hydroxybutyrate attenuated reactive oxygen species production and alleviated apoptosis induced by oxidative stress in cardiomyocytes, suggesting that the accumulation of $\beta$-hydroxybutyrate occurs as a compensatory response against oxidative stress in failing heart [13]. Very recently, ketone body infusion with 3-hydroxybutyrate has been reported to reduce myocardial glucose uptake and increase myocardial blood flow in healthy humans, suggesting that ketone bodies are important cardiac fuels and vasodilators, which may have therapeutic potentials [14]. However, further well-planned studies, preferably by using a greater number of subjects, should be performed to understand an association between ketone body metabolism and heart failure.

\section{Conflicts of Interest}

The authors declare that they have no conflicts of interest concerning this article.

\section{References}

1. Kutoh E. Effect of Tofogliflozin on Cardiac Function: Potential Link Between Ketone Bodies and the Heart. J Endocrinol Metab. 2017;7(3):94-97.

2. Zinman B, Wanner C, Lachin JM, Fitchett D, Bluhmki E, Hantel S, Mattheus M, et al. Empagliflozin, Cardiovascular Outcomes, and Mortality in Type 2 Diabetes. N Engl J Med. 2015;373(22):2117-2128.

3. Kosiborod M, Cavender MA, Fu AZ, Wilding JP, Khunti K, Holl RW, Norhammar A, et al. Lower Risk of Heart Failure and Death in Patients Initiated on Sglt-2 Inhibi- 
tors Versus Other Glucose-Lowering Drugs: The CvdReal Study. Circulation. 2017 May 18.

4. Joubert M, Jagu B, Montaigne D, Marechal X, Tesse A, Ayer A, Dollet L, et al. The Sodium-Glucose Cotransporter 2 Inhibitor Dapagliflozin Prevents Cardiomyopathy in a Diabetic Lipodystrophic Mouse Model. Diabetes. 2017;66(4):1030-1040.

5. Habibi J, Aroor AR, Sowers JR, Jia G, Hayden MR, Garro M, Barron B, et al. Sodium Glucose Transporter 2 (Sglt2) Inhibition with Empagliflozin Improves Cardiac Diastolic Function in a Female Rodent Model of Diabetes. Cardiovasc Diabetol. 2017;16(1):9.

6. Marx N, McGuire DK. Sodium-Glucose Cotransporter-2 Inhibition for the Reduction of Cardiovascular Events in High-Risk Patients with Diabetes Mellitus. Eur Heart J. 2016;37(42):3192-3200.

7. Kashiwagi A, Maegawa H. Metabolic and Hemodynamic Effects of Sodium-Dependent Glucose Cotransporter 2 Inhibitors on Cardio-Renal Protection in the Treatment of Patients with Type 2 Diabetes Mellitus. J Diabetes Investig. 2017 Feb 8.

8. Taylor SI, Blau JE, Rother KI. Sglt2 Inhibitors May Predispose to Ketoacidosis. J Clin Endocrinol Metab. 2015;100(8):2849-2852.

9. Ferrannini E, Mark M, Mayoux E. Cv Protection in the
Empa-Reg Outcome Trial: A "Thrifty Substrate" Hypothesis. Diabetes Care. 2016;39(7):1108-1114.

10. Martens P, Mathieu C, Verbrugge FH. Promise of Sglt2 Inhibitors in Heart Failure: Diabetes and Beyond. Curr Treat Options Cardiovasc Med. 2017;19(3):23.

11. Mudaliar S, Alloju S, Henry RR. Can a Shift in Fuel Energetics Explain the Beneficial Cardiorenal Outcomes in the Empa-Reg Outcome Study? A Unifying Hypothesis. Diabetes Care. 2016;39(7):1115-1122.

12. Bedi KC, Jr., Snyder NW, Brandimarto J, Aziz M, Mesaros C, Worth AJ, Wang LL, et al. Evidence for Intramyocardial Disruption of Lipid Metabolism and Increased Myocardial Ketone Utilization in Advanced Human Heart Failure. Circulation. 2016;133(8):706-716.

13. Nagao M, Toh R, Irino $Y$, Mori $T$, Nakajima H, Hara $\mathrm{T}$, Honjo $\mathrm{T}$, et al. Beta-Hydroxybutyrate Elevation as a Compensatory Response against Oxidative Stress in Cardiomyocytes. Biochem Biophys Res Commun. 2016;475(4):322-328.

14. Gormsen LC, Svart M, Thomsen HH, Sondergaard E, Vendelbo MH, Christensen N, Tolbod LP, et al. Ketone Body Infusion with 3-Hydroxybutyrate Reduces Myocardial Glucose Uptake and Increases Blood Flow in Humans: A Positron Emission Tomography Study. J Am Heart Assoc. 2017;6(3). 